PROCEEDINGS OF THE

AMERICAN MATHEMATICAL SOCIETY

Volume 132, Number 6, Pages 1755-1760

S 0002-9939(03)07355-6

Article electronically published on December 22, 2003

\title{
BACKWARD UNIQUENESS FOR SOLUTIONS OF LINEAR PARABOLIC EQUATIONS
}

\author{
IGOR KUKAVICA
}

(Communicated by David S. Tartakoff)

\begin{abstract}
We address the backward uniqueness property for the equation $u_{t}-\Delta u=w_{j} \partial_{j} u+v u$ in $\mathbb{R}^{n} \times\left(T_{0}, 0\right]$. We show that under rather general conditions on $v$ and $w,\left.u\right|_{t=0}=0$ implies that $u$ vanishes to infinite order for all points $(x, 0)$. It follows that the backward uniqueness holds if $w=0$ and $v \in L^{\infty}\left(\left[0, T_{0}\right], L^{p}\left(\mathbb{R}^{n}\right)\right)$ when $p>n / 2$. The borderline case $p=n / 2$ is also covered with an additional continuity and smallness assumption.
\end{abstract}

\section{INTRODUCTION}

A question of a solution semigroup of an equation on $\mathbb{R}^{n}$,

$$
u_{t}-\Delta u+f(x, u, \nabla u)=0,
$$

being one-to-one is clearly reduced to the following problem: If a solution $u$ of

$$
u_{t}-\Delta u+w_{j} \partial_{j} u+v u=0
$$

on $\mathbb{R}^{n} \times\left(-T_{0}, 0\right]$ vanishes for $t=0$, does it necessarily vanish for $t<0$ ? This question of backward uniqueness was raised by Lax $[\mathrm{L}$ and addressed by various authors $([\mathrm{L},[\mathrm{P},[\mathrm{LP}, \overline{\mathrm{G}}$, [AN1, $[\mathrm{AN2}])$. The approach by weighted inequalities, resembling the Carleman inequality method, has been employed by Lees and Protter $[\mathrm{P},[\mathrm{LP}$. In particular, the backward uniqueness property was proved for the differential inequality

$$
\left(u_{t}-\Delta u\right)^{2} \leq \frac{C}{t^{2}} u^{2}+C|\nabla u|^{2} .
$$

When comparing with the equation (1.1), this covers the case $v \in L^{\infty}\left(\left(T_{0}, 0\right)\right.$, $\left.L^{p_{1}}\left(\mathbb{R}^{n}\right)\right)$ and $w \in L^{\infty}\left(\left(T_{0}, 0\right), L^{p_{2}}\left(\mathbb{R}^{n}\right)\right)$ when $p_{1} \geq n$ and $p_{2}=\infty$. The logarithmic convexity approach, introduced by Agmon and Nirenberg AN1, AN2 and used in $[\mathrm{A}],[\mathrm{BT}, \mathrm{G}]$, is a lot simpler. However, it leads to the same range of exponents $p_{1}, p_{2}$ (cf. [G]). Based on forward uniqueness results, it seems that backward uniqueness should hold when $p_{1} \geq n / 2$ and $p_{2} \geq n$ with sufficiently small norms when $p_{1}=n / 2$ and $p_{2}=n$.

Recently, Escauriaza and Vega (cf. [E], [EV]) showed that if $u$, a solution of (1.1), vanishes of infinite order in space-time at $(x, t)=(0,0)$, and if $w=0$ and $v \in L^{\infty}\left(\left(T_{0}, 0\right), L^{p_{1}}\left(\mathbb{R}^{n}\right)\right)$ where $p_{1} \geq n / 2$ (with sufficiently small norm when $p_{1}=$

Received by the editors February 7, 2003.

2000 Mathematics Subject Classification. Primary 35K15.

Key words and phrases. Backward uniqueness, parabolic equation, parabolic inequalities, backward stability. 
$n / 2)$, then it vanishes identically for $t \in\left(T_{0}, 0\right)$. If one tries to apply this result to backward uniqueness, then one runs into a problem of showing that $u(\cdot, 0) \equiv 0$ implies that $u$ vanishes of infinite order at $(x, 0)$ for every $x \in \mathbb{R}^{n}$. This is the main result of our note. In Theorem 2.1 we namely prove that if $u$ vanishes for $t=0$, and if $p_{1}>n / 2$ and $p_{2}>n$, then $u$ vanishes of infinite order at every point $(x, 0)$ $\left(x \in \mathbb{R}^{n}\right)$. This shows that the backward uniqueness property holds for all $p_{1}>n / 2$ and $w=0$. As for the borderline case, it holds if the corresponding norm of $v$ is sufficiently small and $v(\cdot, t)$ is continuous as an $L^{n / 2}$-valued function.

\section{THE MAIN RESULTS}

Fix any $T_{0}<0$ and $n \in \mathbb{N}$. Let

$$
u \in C^{1}\left(\left[T_{0}, 0\right], H^{1}\left(\mathbb{R}^{n}\right)\right) \cap C\left(\left[T_{0}, 0\right], H^{2}\left(\mathbb{R}^{n}\right)\right)
$$

be a solution of

$$
u_{t}-\Delta u=f .
$$

Note that $f \in C\left(\left[T_{0}, 0\right], L^{2}\left(\mathbb{R}^{n}\right)\right)$. On $f$, we impose the following assumptions:

(A1) $\|f\|_{L^{2}} \leq K_{1}\|\Delta u\|_{L^{2}}+K_{2}\|u\|_{L^{2}}$ for every $t \in\left[T_{0}, 0\right)$, where $\|\cdot\|_{L^{2}}=$ $\|\cdot\|_{L^{2}\left(\mathbb{R}^{n}\right)}$

(A2) $-(f, u) \leq K_{3}\|\nabla u\|_{L^{2}}^{2}+K_{4}\|u\|_{L^{2}}^{2}$ for every $t \in\left[T_{0}, 0\right)$,

(A3) $(f, u) \leq K_{5}\|\nabla u\|_{L^{2}}^{2}+K_{6}\|u\|_{L^{2}}^{2}$ for every $t \in\left[T_{0}, 0\right)$

where $K_{1}, K_{2}, K_{3}, K_{4}, K_{5}$, and $K_{6} \geq 0$. In the logarithmic convexity approach to uniqueness ( $\mathrm{A}$, [AN1], AN2, $\mathrm{G}]$ ), the Dirichlet quotient

$$
Q(t)=\frac{\|\nabla u(t)\|_{L^{2}}^{2}}{\|u(t)\|_{L^{2}}^{2}}
$$

plays a distinguished role; here and in the sequel, we write $u(t)=u(\cdot, t)$. Also, as usual, the symbol $C$ denotes a sufficiently large universal constant depending only on $n$; it has a different value at each occurrence.

The following is our main result.

Theorem 2.1. Let $u$ be as above. Assume $u(0) \equiv 0$ and $u(t) \not \equiv 0$ for $t \in\left[T_{0}, 0\right)$. If $K_{1}<1$ and $K_{5}<1$, then

$$
\|u(t)\|_{L^{2}} \leq\left\|u\left(T_{1}\right)\right\|_{L^{2}}\left|\frac{t}{T_{1}}\right|^{M} \exp \left(K_{6}\left(t-T_{1}\right)\right)
$$

for $t \in\left[T_{1}, 0\right)$, where

$$
T_{1}=\max \left\{T_{0},-\frac{1-K_{1}}{C K_{1} K_{2}}\right\}
$$

and

$$
M=\frac{\left(1-K_{5}\right)\left(1-K_{1}\right)}{C K_{1}^{2}} .
$$

Of particular interest is the equation of the form

$$
u_{t}-\Delta u=w_{j} \partial_{j} u+v u
$$

where $u$ is as in (2.1). 
Corollary 2.2. Assume $u$, which solves (2.3), satisfies $u(0) \equiv 0$ and $u(t) \not \equiv 0$ for $t \in\left[T_{0}, 0\right)$. Assume $w_{j} \in C\left(\left[T_{0}, 0\right], L^{n}\left(\mathbb{R}^{n}\right)\right.$ ) and $v \in C\left(\left[T_{0}, 0\right], L^{n / 2}\left(\mathbb{R}^{n}\right)\right.$ ) (mere continuity at 0 and $v \in L^{\infty}\left(\left[T_{0}, 0\right], L^{n / 2}\left(\mathbb{R}^{n}\right)\right)$ suffice $)$. Then

$$
\|u(t)\|_{L^{2}}=o\left(|t|^{m}\right), \quad \text { as } t \rightarrow 0-
$$

i.e.,

$$
\lim _{t \rightarrow 0-} \frac{\|u(t)\|_{L^{2}}}{|t|^{m}}=0
$$

for every $m \in \mathbb{N}$.

Proof of Corollary 2.2. Let $\epsilon>0$ be arbitrary. Then there exist $T_{1}<0$ and decompositions $w_{j}=w_{j 1}+w_{j 2}$ and $v=v_{1}+v_{2}$ so that $w_{j 1}, w_{j 2} \in C\left(\left[T_{1}, 0\right], L^{n}\left(\mathbb{R}^{n}\right)\right)$ and $v_{1}, v_{2} \in C\left(\left[T_{1}, 0\right], L^{n / 2}\left(\mathbb{R}^{n}\right)\right)$ with

$$
\left\|w_{j 1}\right\|_{L^{\infty}\left(\left[T_{1}, 0\right], L^{n}\left(\mathbb{R}^{n}\right)\right)},\left\|v_{1}\right\|_{L^{\infty}\left(\left[T_{1}, 0\right], L^{n / 2}\left(\mathbb{R}^{n}\right)\right.} \leq \epsilon
$$

and

$$
w_{j 2}, v_{2} \in L^{\infty}\left(\left[T_{1}, 0\right], L^{\infty}\left(\mathbb{R}^{n}\right)\right) .
$$

Then, by Sobolev imbedding, (A1), (A2), and (A3) hold with

$$
K_{1}=K_{3}=K_{5}=C \epsilon
$$

where $f=w_{j} \partial_{j} u+v u$, where $C$ depends only on $n$, and where $K_{2}, K_{4}$, and $K_{6}$ are some positive constants. If $K_{1}, K_{5}<1$ (which holds if $\epsilon<1 / C$ ), then Theorem 2.1 implies

$$
\|u(t)\|_{L^{2}}=\mathcal{O}\left(|t|^{(1-C \epsilon) / C^{2} \epsilon^{2}}\right), \quad \text { as } t \rightarrow 0-
$$

and Corollary 2.2 indeed follows from Theorem 2.1

Corollary 2.3. Assume that $u$, which solves (2.3) on $t \in\left[T_{0}, 0\right)$, satisfies $u(0) \equiv 0$. Assume $w_{j}=0$ and $v \in C\left(\left[T_{0}, 0\right], L^{n / 2}\left(\mathbb{R}^{n}\right)\right)$ (mere continuity at 0 suffices) with $\|v\|_{L^{\infty}\left(\left[T_{0}, 0\right], L^{n / 2}\left(\mathbb{R}^{n}\right)\right)}<\epsilon$ where $\epsilon=\epsilon(n)>0$. Then $u(t) \equiv 0$ for $t \in\left[T_{0}, 0\right]$.

Proof of Corollary [2.3] The previous corollary implies that

$$
\|u(t)\|_{L^{2}}=\mathcal{O}\left(|t|^{m}\right), \quad \text { as } t \rightarrow 0-
$$

for all $m \in \mathbb{N}$. Under this assumption, a result of Escauriaza and Vega [EV] implies that $u(t)=0$ for all $t \in\left[T_{0}, 0\right]$.

The same conclusion holds if $w_{j}=0$ and $v \in L^{\infty}\left(\left[T_{0}, 0\right], L^{p}\left(\mathbb{R}^{n}\right)\right)$ when $p \in$ $(n / 2, \infty]$; in this case we use [E].

\section{Proof of the main Result}

In this section, we prove Theorem [2.1] We start with a simple lemma.

Lemma 3.1. Let $x \in C^{1}\left(\left[T_{0}, 0\right),[0, \infty)\right)$ be such that

$$
\dot{x} \leq A x^{\alpha}+B
$$

on $\left[T_{0}, 0\right)$ where $T_{0}<0, A>0, B \geq 0$, and $\alpha>1$. Assume that $x$ is unbounded on $\left[T_{0}, 0\right)$. Then for

$$
t \in\left[-\frac{1}{4 B^{(\alpha-1) / \alpha} A^{1 / \alpha}}, 0\right) \cap\left[T_{0}, 0\right),
$$


we have

$$
x(t)>\frac{1}{2^{(\alpha+1) /(\alpha-1)}|t|^{1 /(\alpha-1)} A^{1 /(\alpha-1)}} .
$$

Proof of Lemma [3.1. Denote by $\gamma(t)$ the right-hand side of (3.3). Assume, contrary to the assertion, that there exists $t$ satisfying (3.2) such that

$$
x(t) \leq \gamma(t) .
$$

Using the Gronwall lemma, we shall show that (3.4) implies that

$$
x(\tau) \leq 2 \gamma(t), \quad \tau \in(t, 0)
$$

and this contradicts the fact that $x$ is unbounded. It remains to be shown that (3.4), together with $x \in C^{1}\left(\left[T_{0}, 0\right),[0, \infty)\right)$ and (3.1) on $[t, 0)$, implies (3.5). Assume this is not true. Then we can find

$$
\tau_{0}=\min \{\tau \in(t, 0): x(\tau)=2 \gamma(t)\} .
$$

Note that $t<\tau_{0}<0$. Then

$$
\dot{x}(\tau) \leq A x(\tau)^{\alpha}+B \leq 2^{\alpha} A \gamma(t)^{\alpha}+B
$$

for $\tau \in\left[t, \tau_{0}\right]$, which implies that

$$
x\left(\tau_{0}\right) \leq \gamma(t)+\left(2^{\alpha} A \gamma(t)^{\alpha}+B\right)\left(\tau_{0}-t\right)<\gamma(t)+\left(2^{\alpha} A \gamma(t)^{\alpha}+B\right)|t| .
$$

Using the definition of $\gamma(t)$ and (3.2), one can check that $2^{\alpha} A \gamma(t)^{\alpha} \leq \gamma(t) / 2|t|$ and $B \leq \gamma(t) / 2|t|$. We get $x\left(\tau_{0}\right)<2 \gamma(t)$, which is a contradiction.

Proof of Theorem [2.1. Assume that $u(0) \equiv 0$ and $u(t) \not \equiv 0$ for $t \in\left[T_{0}, 0\right)$. First, we claim that $Q(t)$ is unbounded on $\left[T_{0}, 0\right)$. From (2.2) and (A2), we deduce

$$
\begin{aligned}
& \frac{1}{2} \frac{d}{d t}\|u\|_{L^{2}}^{2}+Q(t)\|u\|_{L^{2}}^{2}=(f, u) \\
& \quad \geq-K_{3}\|\nabla u\|_{L^{2}}^{2}-K_{4}\|u\|_{L^{2}}^{2}=-\|u\|_{L^{2}}^{2}\left(K_{3} Q(t)+K_{4}\right) .
\end{aligned}
$$

Integrating this inequality, we get

$$
\|u(t)\|_{L^{2}} \geq\left\|u\left(T_{0}\right)\right\|_{L^{2}} \exp \left(-\left(1+K_{3}\right) \int_{T_{0}}^{t} Q(\tau) d \tau-K_{4}\left(t-T_{0}\right)\right)
$$

provided $t \in\left[T_{0}, 0\right)$. If $Q(t)$ were bounded, we would have $u(0) \not \equiv 0$, which contra$\operatorname{dicts} u(0) \equiv 0$.

Next, we claim that

$$
Q(t) \geq \frac{1-K_{1}}{8 K_{1}^{2}|t|}, \quad t \in\left[T_{1}, 0\right)
$$

where

$$
T_{1}=\max \left\{T_{0},-\frac{1-K_{1}}{4 K_{1} K_{2}}\right\} .
$$

By AN1 and AN2, we have an identity

$$
\frac{1}{2} Q^{\prime}(t)+\|(A-Q(t) I) v\|_{L^{2}}^{2}=\frac{1}{\|u\|_{L^{2}}^{2}}(f,(A-Q(t) I))
$$


where $A=-\Delta$ and $v=u /\|u\|_{L^{2}}$. Therefore, by assumption (A1),

$$
\begin{aligned}
& \frac{1}{2} Q^{\prime}(t)+\|(A-Q(t) I) v\|_{L^{2}}^{2} \\
& \quad \leq \frac{\|f\|_{L^{2}}}{\|u\|_{L^{2}}}\|(A-Q(t) I) v\|_{L^{2}} \leq\left(K_{1}\|A v\|_{L^{2}}+K_{2}\right)\|(A-Q(t) I) v\|_{L^{2}}
\end{aligned}
$$

since $\|v\|_{L^{2}}=1$. Now, using

$$
\|A v\|_{L^{2}} \leq\|(A-Q(t) I) v\|_{L^{2}}+Q(t)
$$

we obtain

$$
\begin{aligned}
& \frac{1}{2} Q^{\prime}(t)+\|(A-Q(t) I) v\|_{L^{2}}^{2} \\
& \quad \leq K_{1}\|(A-Q(t) I) v\|_{L^{2}}^{2}+K_{1} Q(t)\|(A-Q(t) I) v\|_{L^{2}}+K_{2}\|(A-Q(t) I) v\|_{L^{2}} .
\end{aligned}
$$

Since $K_{1}<1$, we get by the Cauchy-Schwarz inequality,

$$
Q^{\prime}(t) \leq \frac{K_{1}^{2}}{1-K_{1}} Q(t)^{2}+\frac{K_{2}^{2}}{1-K_{1}} .
$$

By Lemma 3.1

$$
Q(t) \geq \frac{1-K_{1}}{8 K_{1}^{2}|t|}=-\frac{1-K_{1}}{8 K_{1}^{2} t}
$$

for $t \in\left[T_{1}, 0\right)$, where $T_{1}$ is as in (3.7), and the claim is established. In order to conclude the proof, we use the first equality in (3.6) and assumption (A3) to obtain

$$
\frac{1}{2} \frac{d}{d t}\|u\|_{L^{2}}^{2}+Q(t)\|u\|_{L^{2}}^{2} \leq\left(K_{5} Q(t)+K_{6}\right)\|u\|_{L^{2}}^{2}
$$

from which

$$
\frac{d}{d t}\|u\|_{L^{2}}+\left(\left(1-K_{5}\right) Q(t)-K_{6}\right)\|u\|_{L^{2}} \leq 0 .
$$

Since $K_{5}<1$, we get

$$
\frac{d}{d t}\|u\|_{L^{2}}+\left(-\frac{\left(1-K_{5}\right)\left(1-K_{1}\right)}{8 K_{1}^{2} t}-K_{6}\right)\|u\|_{L^{2}} \leq 0
$$

for $t \in\left[T_{1}, 0\right)$. Integrating this inequality, we obtain

$$
\|u(t)\|_{L^{2}} \leq\left\|u\left(T_{1}\right)\right\|_{L^{2}} \exp \left(\frac{\left(1-K_{5}\right)\left(1-K_{1}\right)}{8 K_{1}^{2}} \log \left|\frac{t}{T_{1}}\right|+K_{6}\left(t-T_{1}\right)\right)
$$

for all $t \in\left[T_{1}, 0\right)$.

\section{ACKNOWLEDGMENT}

This work was supported in part by the NSF grant DMS-0072662 while the author was an Alfred P. Sloan fellow. 


\section{REFERENCES}

[A] S. Agmon, Unicité et convexité dans les problèmes différentiels, Séminaire de Mathématiques Supérieures, No. 13 (Eté, 1965), Les Presses de l’Université de Montréal, Montreal, Quebec, 1966. MR 40:6025

[AN1] S. Agmon and L. Nirenberg, Properties of solutions of ordinary differential equations in Banach space, Comm. Pure Appl. Math. 16 (1963), 121-239. MR 27:5142

[AN2] S. Agmon and L. Nirenberg, Lower bounds and uniqueness theorems for solutions of differential equations in a Hilbert space, Comm. Pure Appl. Math. 20 (1967), 207-229. MR 34:4665

[BT] C. Bardos and L. Tartar, Sur l'unicité rétrograde des équations paraboliques et quelques questions voisines, Arch. Rational Mech. Anal. 50 (1973), 10-25. MR 49:3281]

[E] L. Escauriaza, Carleman inequalities and the heat operator, Duke Math. J. 104 (2000), 113-127. MR 2001m:35135

[EV] L. Escauriaza and L. Vega, Carleman inequalities and the heat operator. II, Indiana Univ. Math. J. 50 (2001), 1149-1169. MR 2003b:35088

[G] J.-M. Ghidaglia, Some backward uniqueness results, Nonlinear Anal. 10 (1986), 777-790. MR 87m:34083

[L] P. D. Lax, A stability theorem for solutions of abstract differential equations, and its application to the study of the local behavior of solutions of elliptic equations, Comm. Pure Appl. Math. 9 (1956), 747-766. MR 19:281a

[LP] M. Lees and M. H. Protter, Unique continuation for parabolic differential equations and inequalities, Duke Math. J. 28 (1961), 369-382. MR 25:4254

[P] M. H. Protter, Properties of solutions of parabolic equations and inequalities, Canad. J. Math. 13 (1961), 331-345. MR 27:3943

Department of Mathematics, University of Southern California, Los Angeles, CalIFORNIA 90089

E-mail address: kukavica@usc.edu 